\title{
Biological Grandfather
}

National Cancer Institute

\section{Source}

National Cancer Institute. Biological Grandfather. NCI Thesaurus. Code C96573.

A male relative who is the biological father of either the biological mother or the biological father. 